\title{
Anordnungsmodell: \\ Chronologie einer Kampfansage
}

\section{Stellungnahme der ASP zum Verordnungsentwurf zur Neuregelung der Psychotherapie}

\section{Marianne Roth}

Der Bundesrat hat am 26. Juni 2019 die Vernehmlassung zur Neuregelung der psychologischen Psychotherapie im Rahmen der obligatorischen Krankenpflegeversicherung (OKP) eröffnet. Die seit Langem fällige Änderung des Delegations- ins Anordnungsmodell (für einen Vergleich siehe die Tabelle) geht für die ASP in die richtige Richtung. Aus Praxissicht gibt es jedoch einiges an Verbesserungspotenzial.

Mit einer Online-Umfrage haben wir die Meinungen unserer Mitglieder eingeholt und diese in die Stellungnahme einfliessen lassen. Die über 1000 Kommentare, die mit den Antworten geliefert wurden, setzten zusätzliche Schwergewichte und waren eine sinnvolle Ergänzung zu den rein numerischen Ergebnissen.

Erfreulicher Nebeneffekt ist auch, dass die Stellungnahme der ASP nicht im stillen Kämmerlein entstanden ist, sondern gemeinsam mit den Partnerverbänden FSP und SBAP ausdiskutiert und verfasst wurde. Das Resultat ist eine einheitliche Position, die die drei Verbände gemeinsam vertreten und pro Verband eingereicht haben.

\section{Eckpunkte der Vorlage, die die ASP klar stützt}

Die ASP ist vollumfänglich einverstanden mit den Zielen der Neuregelung der psychologischen Psychotherapie. Es braucht einen verbesserten Zugang zur Psychotherapie, speziell auch in Krisen- und Notfallsituationen. Durch die Anordnung seitens der Grundversorger werden bestehende Hürden in der Versorgung von psychisch Kranken abgebaut. Weiter stützen wir klar die durch den Modellwechsel erwartete Verbesserung der Qualität der Leistungserbringung durch die Verbindlichkeit des Psychologieberufegesetzes, das seit 18. März 2011 in Kraft ist.

Ebenfalls einverstanden ist die ASP mit den Anordnungsvoraussetzungen respektive dem Vorschlag in Bezug auf die anordnungsberechtigen Ärzt*innen. Es sollen nicht nur Fachärzt*innen der Psychiatrie Anordnungsbefugnis erhalten, sondern auch Fachärzt*innen zusätzlicher Kategorien. Dies sichert den niederschwelligen
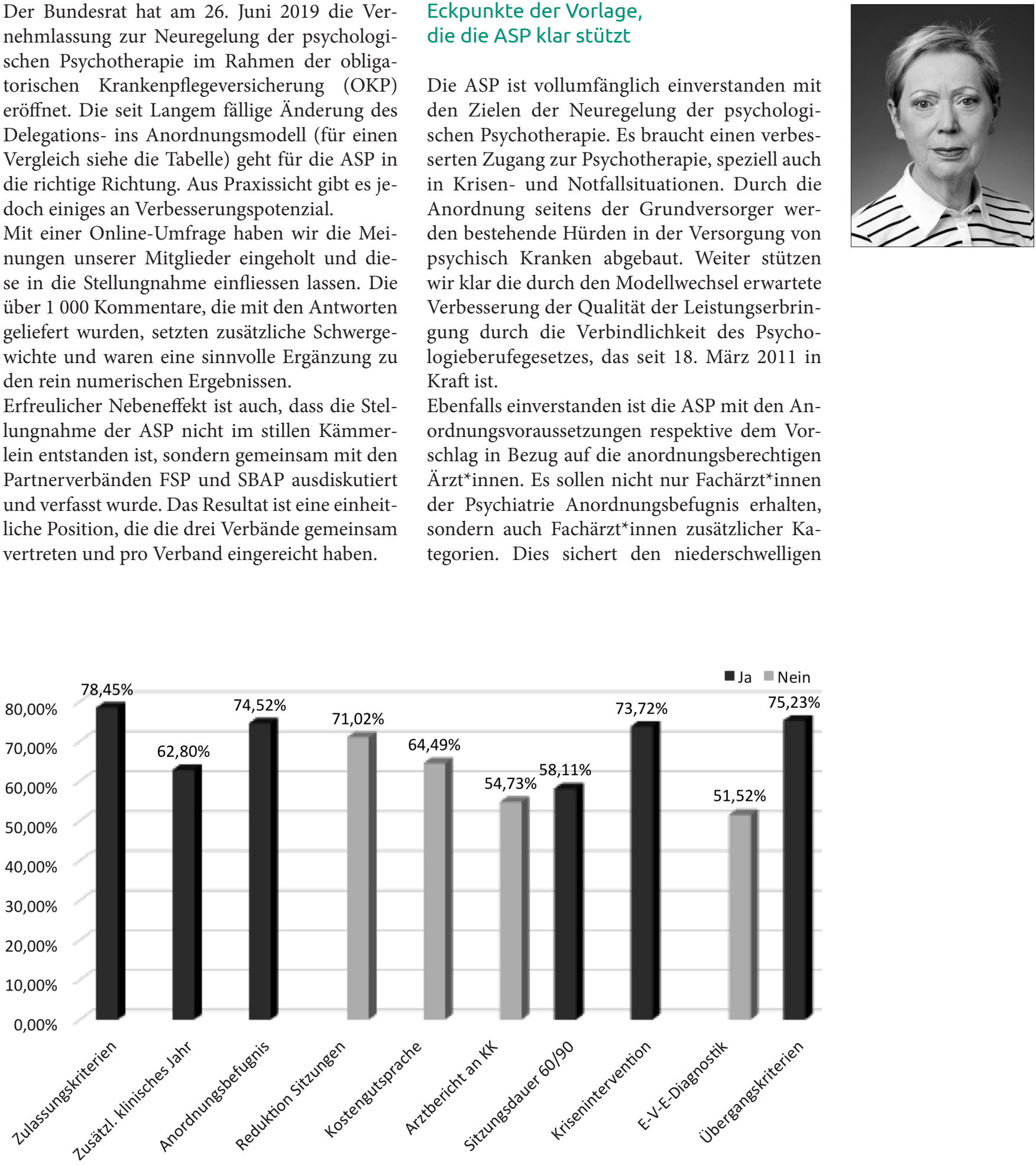

Abb. 1: Mitgliederbefragung zum Anordnungsmodell 
Zugang zur Psychotherapie, was die rechtzeitige Versorgung der Patient*innen erhöht und durch weniger Chronifizierung und stationäre Aufenthalte langfristig Kosten im Gesundheitssystem einspart.

\section{Inhalte der Vorlage, die die ASP ablehnt und deren Anpassung sie fordert}

Die ASP lehnt die Beschränkung auf 30 Sitzungen pro Anordnung ab. Sie fordert die Beibehaltung der bisherigen Anordnung von 40 Sitzungen, da sich diese Praxis bewährt hat und die Beschränkung auf 30 Sitzungen den administrativen Aufwand und letztendlich die Kosten steigert. Das vorgesehene Berichtswesen nach 30 Stunden verursacht zudem einen unverhältnismässigen Mehraufwand bei allen Beteiligten. Die langjährige Praxis zeigt, dass Anordnungen nicht unnötig ausgeschöpft werden.

Die stufenweise Anordnung, wonach bereits nach 15 Sitzungen anordnenden Ärzt*innen ein Bericht vorgelegt werden muss, um nochmals 15 Sitzungen angeordnet zu erhalten, lehnt die ASP strikt ab. Dieses Ansinnen ist kostenstei- gernd, ohne zusätzlichen Nutzen und benachteiligt psychologische Psychotherapeut*innen gegenüber den ärztlichen Leistungserbringern. Solch eine Ungleichbehandlung ist sachlich nicht gerechtfertigt. Sie führt lediglich zu einer Überbelastung und zum Nachteil hinsichtlich der Therapiezeit für die Patient*innen.

Die ASP ist mit dem zusätzlichen klinischen Jahr einverstanden. Es ist jedoch nicht nachvollziehbar, weshalb das zusätzliche klinische Jahr erst nach Abschluss der Weiterbildung erfolgen soll. Es muss daher zwingend möglich sein, dieses nicht nur nach, sondern bereits während der Weiterbildung $\mathrm{zu}$ absolvieren. Zudem soll es nicht nur unter der Leitung von Fachärzt*innen für Psychiatrie absolviert werden können, sondern auch unter der Leitung eidgenössisch anerkannter Psychotherapeut*innen.

Die ASP ist der Meinung, dass die Sitzungsdauer nicht auf Verordnungsebene gelöst werden kann. Die Sitzungsdauer bei Einzel- und Gruppentherapien muss in den Tarifverträgen geregelt werden. Die ASP verlangt daher die Streichung der Präzisierungen zur Dauer der jeweiligen Sitzungen im Verordnungsvorschlag.

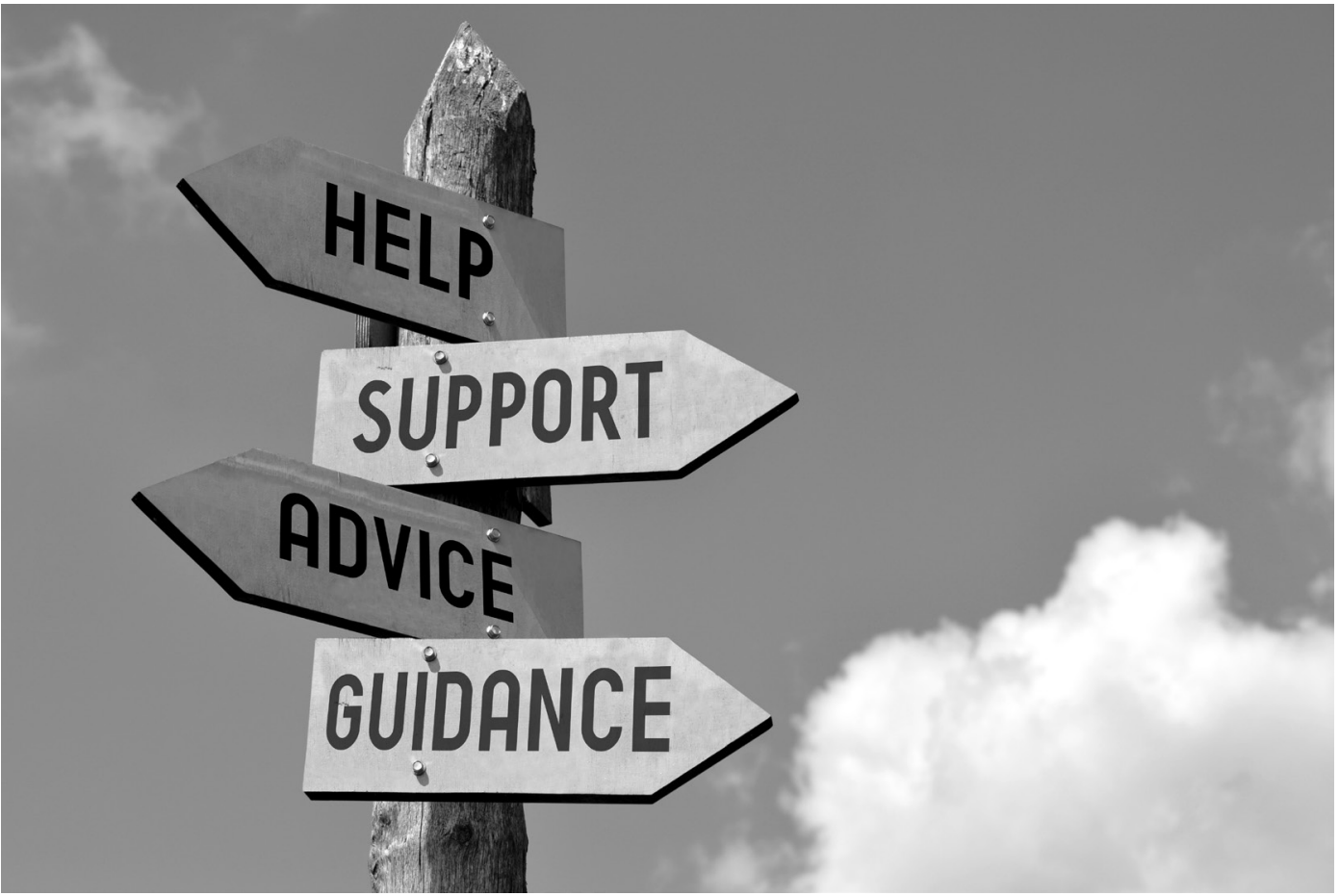


Psychologische Psychotherapeut*innen erbringen ihre Leistungen als Angestellte in einer Arztpraxis unter Aufsicht und in der Verantwortung der/s delegierenden Ärztin/ Arztes. Diese rechnen die Leistung gemäss TARMED Tarif mit der Grundversicherung ab.
Ärzt*innen müssen eine Psychotherapie anordnen. Eidgenössisch anerkannte psychologische Psychotherapeut*innen erbringen die Leistung in der Folge selbstständig und in eigener Verantwortung. Sie rechnen die Leistungen mit der Grundversicherung direkt ab.
Die ASP ist grundsätzlich mit dem Antrag auf Fortsetzung der Therapie durch anordnende Ärzt ${ }^{*}$ innen einverstanden - dies allerdings erst nach 40 Sitzungen. Der Bericht, der dazu abgeliefert werden soll, muss jedoch zwingend von den behandelnden Psychotherapeut*innen verfasst, unterzeichnet und verrechnet werden.

Die ASP ist gegen die Einführung einer Einstiegs-, Verlaufs- und Erfolgsdiagnostik, wie sie im Verordnungsvorschlag definiert ist. Die Einführung einer solchen Diagnostik für alle Patient*innen würde eine riesige Datenmenge kreieren, deren technische wie auch inhaltliche Verarbeitung und die damit verbundenen Kosten unverhältnismässig wären. Stattdessen verlangt die ASP die Prüfung der Tauglichkeit und Praktikabilität einer solchen Diagnostik an einem Modellprojekt. Hernach kann ein Stichprobenverfahren implementiert werden.

Ergänzungen, die im Verordnungsentwurf fehlen oder zu wenig berücksichtigt sind und die die ASP vorschlägt

Psychotherapeut*innen in Ausbildung werden im Verordnungsentwurf zu wenig berücksichtigt. Aus Gründen der Qualitätssicherung stützen wir die vorgeschlagene Regelung, dass Psycholog ${ }^{\star}$ innen in Weiterbildung zu Psychotherapeut*innen nicht zur selbstständigen Abrechnung über die Krankenkasse zugelassen sind. Die ASP verlangt jedoch, dass die berufliche Ausbildung von Psychotherapeut ${ }^{*}$ innen gesichert ist, und wünscht sich die Zusammenarbeit mit den Kantonen für die langfristige Sicherstellung der Ausbildungsplätze. Ob in der Zukunft eine Praxisassistenz analog den $\ddot{\text { rzzt }}{ }^{*}$ innen angezeigt ist, was eine Verrechenbarkeit in der OKP nach sich ziehen müsste, muss überprüft werden. Dies gehört aber in die Weiterbildungsregularien. Es soll analog der ärztlichen Regelung (TARMED Rahmenvertrag Artikel 8) auch für die psychologische Psychotherapie möglich sein.

Mit der aktuellen Vorlage ist unklar, wie die zukünftige Situation angestellter Psychotherapeut*innen in privaten und öffentlichen Organisationen geregelt ist. Aus Sicht der ASP braucht es weiterhin die Möglichkeit, psychotherapeutische Leistungen in einem Anstellungsverhältnis zu erbringen.

Die ASP unterstützt grundsätzlich Massnahmen, die ungerechtfertigten Mengenausweitungen im Gesundheitswesen entgegenwirken. Liegt das Wachstum bei Neuzulassungen in einem Kanton höher als im schweizerischen Durchschnitt, kann sich die ASP eine vergleichbare Lösung wie beim Vernehmlassungsentwurf des indirekten Gegenvorschlags der Pflegeinitiative vorstellen. Psychotherapie basiert auf Sprache. Die ASP fordert für Personen mit ausländischem Diplom als Zulassungskriterium für die Sprachregion, in der praktiziert werden soll, einen Nachweis der Sprachkompetenz auf Niveau C1 der jeweiligen Schweizer Amtssprache.

Marianne Roth ist Geschäftsleiterin der ASP. 